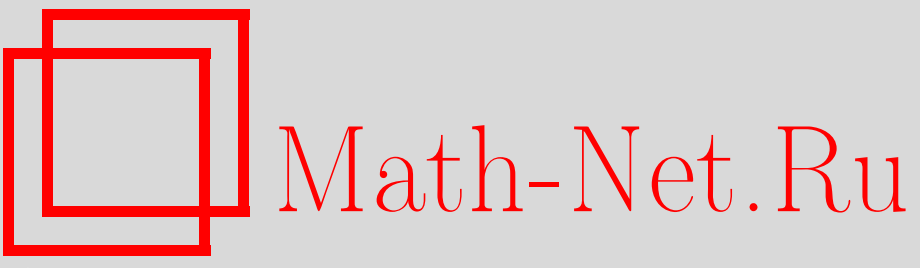

G. M. Rozenblat, Dynamics of non-holonomic models of wheel carriage, Vestn. Udmurtsk. Univ. Mat. Mekh. Komp. Nauki, 2008, Issue 3, 99-108

Use of the all-Russian mathematical portal Math-Net.Ru implies that you have read and agreed to these terms of use

http: //www . mathnet.ru/eng/agreement

Download details:

IP : 107.22 .136 .117

April 26, 2023, 03:11:39 
УДК 531.01

\section{(c) Г. М. Розенблат}

\section{К ДИНАМИКЕ НЕГОЛОНОМНЫХ МОДЕЛЕЙ КОЛЕСНЫХ ЭКИПАЖЕЙ}

В работе рассматриваются две задачи неголономной механики: 1) движение без проскальзывания колесной пары (два диска, свободно укрепленных на оси) по наклонной плоскости в поле силы тяжести, и 2) движение плоской колесной модели типа скейтборда.

Ключевые слова: неголономная связь, основные теоремы динамики, колесный экипаж, скейтборд.

\section{Введение}

При составлении уравнений движения используются основные теоремы динамики и кинематические соотношения, характеризующие неголономные связи. Эта методика дает возможность, наряду с определением движения системы, получать также и выражения для сил, реализующих движение (нормальные и касательные силы реакции опорной плоскости). Таким образом, реализуется известный принцип Ньютона о неразделимости сил и движения. Отметим, что в большинстве недавних работ [1-4] при составлении уравнений движения используются такие известные уравнения, как уравнения Аппеля, Тамена-Больцмана и т. п. Помимо аналитической сложности этих уравнений, в них также отсутствуют реакции связей, что снижает их ценность для практических задач.

\section{$\S 1$. Колесная пара на наклонной плоскости. Уравнения движения, постановка задачи и формулировка результатов}

На рис. 1 изображена колесная пара на наклонной плоскости. Эта конструкция представляет собой два однородных диска, массой $m$ и радиуса $b$ каждый, свободно насаженных на ось $\mathrm{O}_{1} \mathrm{O}_{2}$, представляющую собой однородный стержень массы $M$ и длины $2 l$. Таким образом, диски (колеса) могут свободно и независимо друг от друга вращаться вокруг оси $\mathrm{O}_{1} \mathrm{O}_{2}$ без трения (см. рис. 1).

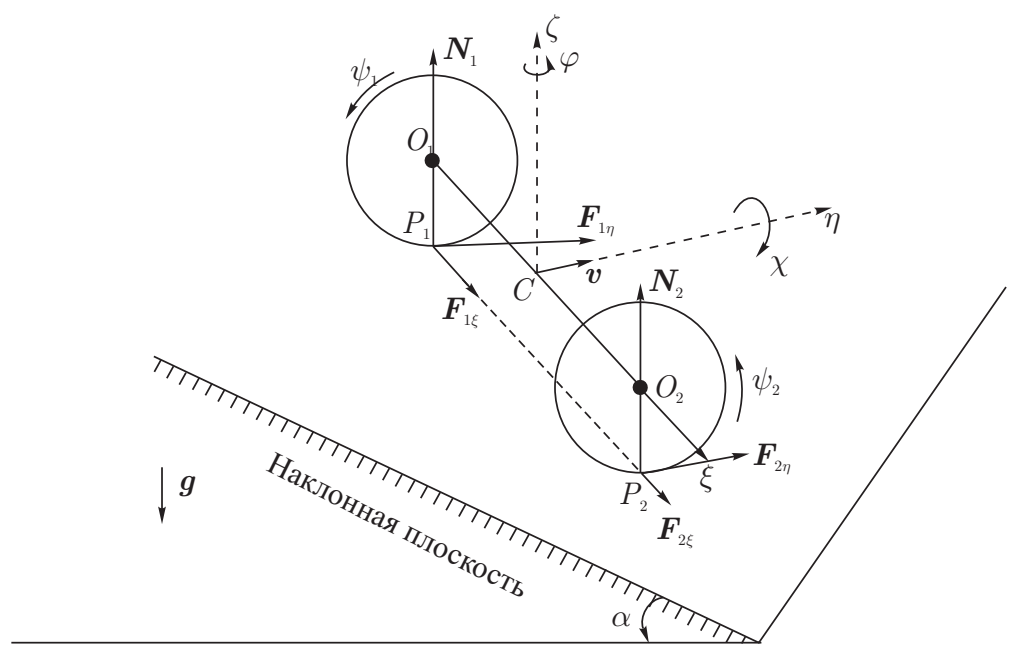

Рис. 1. Колесная пара на наклонной плоскости, в поле силы тяжести. $P_{1}, P_{2}$ - точки контакта колес с опорной плоскостью 
Описанная система движется под действием силы тяжести по шероховатой наклонной плоскости (угол наклона к горизонтали равен $\alpha$ ) так, что в точках контакта колес с плоскостью (точек $P_{1}, P_{2}$, см. рис. 1) не происходит проскальзывания (ни бокового, ни продольного).

Пусть $C$ - центр масс системы (ясно, что он находится в середине отрезка $O_{1} O_{2}$ ), a $C \xi \eta \zeta$ - система координат, жестко связанная с осью $O_{1} O_{2}$, причем (см. рис. 1) $C \xi$ направлена вдоль оси $\mathrm{O}_{1} \mathrm{O}_{2}, \mathrm{C} \zeta$ - нормально наклонной плоскости вверх, а $\mathrm{C \eta}-$ перпендикулярна $C \xi$ и $C \eta$ и образует с ними правую систему координат.

Движение колесной пары происходит под действием силы тяжести и сил реакции плоскости, возникающих в точках контакта $P_{1}$ и $P_{2}$. Эти силы изображены на рис. $1: \mathbf{N}_{1}, \mathbf{N}_{2}-$ нормальные силы реакции, причем значения их проекций на ось $C \zeta$ должны быть неотрицательными $\left(N_{1 \zeta} \geqslant 0, N_{2 \zeta} \geqslant 0\right), \mathbf{F}_{1 \xi}, \mathbf{F}_{2 \xi}$ - поперечные, а $\mathbf{F}_{1 \eta}, \mathbf{F}_{2 \eta}$ - продольные силы сцепления (силы трения покоя). В дальнейшем будем предполагать, что отрыва не происходит и $N_{1 \zeta}, N_{2 \zeta}$ существенно положительные величины. Кроме того, пусть коэффициент трения опорной плоскости настолько велик, что в точках $P_{1}, P_{2}$ отсутствуют проскальзывания, то есть $\mathbf{v}_{P_{1}}=\mathbf{v}_{P_{2}}=0$.

Пусть $\psi_{1}, \psi_{2}$ - собственные углы поворота колес относительно оси $O_{1} O_{2}, \varphi-$ угол поворота оси $\mathrm{O}_{1} \mathrm{O}_{2}$ в наклонной плоскости, отсчитываемый от линии наибольшего ската против часовой стрелки (если смотреть с положительного конца оси $C \zeta$ ).

Таким образом, угловая скорость оси $O_{1} O_{2}$ (а значит, и системы $C \xi \eta \zeta$ ) в проекциях на оси $\xi, \eta, \zeta$ есть:

$$
\boldsymbol{\omega}_{0}=(0,0, \dot{\varphi}) .
$$

Угловая скорость каждого из колес, в проекциях на те же оси:

$$
\boldsymbol{\omega}_{j}=\left(\dot{\psi}_{j}, 0, \dot{\varphi}\right), \quad j=1,2 .
$$

Вычислим скорость точки $O_{1}$, считая ее принадлежащей колесу 1 :

$$
\mathbf{v}_{O_{1}}=\mathbf{v}_{P_{1}}+\left[\boldsymbol{\omega}_{1} \times P_{1} O_{1}\right] .
$$

Отсюда, поскольку $\mathbf{v}_{P_{1}}=0$, получим:

$$
v_{O_{1} \xi}=0, \quad v_{O_{1} \eta}=-b \dot{\psi}_{1}, \quad v_{O_{1} \zeta}=0 .
$$

$\mathrm{C}$ другой стороны, так как точка $O_{1}$ принадлежит оси $O_{1} O_{2}$, то получим:

$$
\mathbf{v}_{O_{1}}=\mathbf{v}_{C}+\left[\omega_{1} \times C O_{1}\right]
$$

или, в проекциях на оси $\xi, \eta, \zeta$, имеем:

$$
v_{O_{1} \xi}=v_{C \xi}, \quad v_{O_{1} \eta}=v_{C \eta}-l \dot{\varphi}, \quad v_{O_{1} \zeta}=v_{C \zeta} .
$$

Из (1.3), (1.4) следуют соотношения

$$
v_{C \xi}=v_{C \zeta}=0, \quad v_{C \eta}-l \dot{\varphi}=-b \psi_{1} .
$$

Аналогичные рассуждения для точки $O_{2}$ приводят к соотношениям:

$$
v_{C \xi}=v_{C \zeta}=0, \quad v_{C \eta}+l \dot{\varphi}=-b \psi_{2} .
$$

Таким образом, условия непроскальзывания приводят к следующим выводам:

1) скорость точки $C$ имеет ненулевую проекцию только на продольную ось $C \eta$, будем обозначать ее в дальнейшем через $v$, то есть

$$
v_{C \xi}=0, \quad v_{C \eta}=v, \quad v_{C \zeta}=0 ;
$$

2) выполняются два соотношения:

$$
\left\{\begin{array}{l}
v+l \dot{\varphi}=-b \dot{\psi}_{2}, \\
v-l \dot{\varphi}=-b \dot{\psi}_{1} .
\end{array}\right.
$$


Переходим к записи уравнений динамики для колесной пары. Уравнения движения центра масс системы в осях $C \xi \eta \zeta$, с учетом (1.1) и (1.5), имеют вид:

$$
\left\{\begin{array}{l}
m_{0}(-v \dot{\varphi})=F_{1} \xi+F_{2} \xi+m_{0} g \sin \alpha \sin \varphi, \\
m_{0} \dot{v}=F_{1} \eta+F_{2} \eta+m_{0} g \sin \alpha \cos \varphi, \\
0=N_{1}+N_{2}-m_{0} g \cos \alpha,
\end{array}\right.
$$

где $m_{0}=M+2 m-$ общая масса системы.

Для составления уравнений изменения кинетического момента в осях $C \xi \eta \zeta$, вычислим сначала сам кинетический момент $\mathbf{K}_{C}$ в этих осях. Обозначим $J_{\xi}, J_{\zeta}$ соответственно осевой и экваториальный моменты инерции колеса $\left(J_{\xi}=m b^{2} / 2, J_{\zeta}=m b^{2} / 4\right), J_{1}$ - момент инерции стержня $O_{1} O_{2}$ относительно оси $C \zeta$ (или $\left.C \eta\right)\left(J_{1}=M l^{2} / 3\right)$. Используя симметрию системы и формулы (1.1) и (1.2) для угловых скоростей, мы получим:

$$
K_{C \xi}=J_{\xi}\left(\dot{\psi}_{1}+\dot{\psi}_{2}\right), \quad K_{C \eta}=0, \quad K_{C \zeta}=J_{0} \dot{\varphi}
$$

где $J_{0}=J_{1}+2 J_{\zeta}+2 m l^{2}$. Отметим, что равенство $K_{C \eta}=0$ следует из того, что нет вращения колесной пары вокруг продольной оси $C \eta$ (то есть колеса не отрываются от опорной плоскости). Используя (1.8) и соотношение (1.1) для угловой скорости системы $C \xi \eta \zeta$, получим уравнения теоремы об изменении кинетического момента $\mathbf{K}_{C}$ в осях $C \xi \eta \zeta$ :

$$
\left\{\begin{array}{l}
J_{\xi}\left(\ddot{\psi}_{1}+\ddot{\psi}_{2}\right)=b\left(F_{1 \eta}+F_{2 \eta}\right), \\
J_{\xi} \dot{\varphi}\left(\dot{\psi}_{1}+\dot{\psi}_{2}\right)=\left(N_{1}-N_{2}\right) l-b\left(F_{1 \xi}+F_{2 \xi}\right), \\
J_{0} \ddot{\varphi}=l\left(F_{2 \eta}-F_{1 \eta}\right) .
\end{array}\right.
$$

Далее запишем уравнения для изменения кинетического момента для каждого из колес в отдельности относительно оси $\xi$, в предположении, что они вращаются свободно на оси $\xi$ и нет поворотов вокруг оси $C \eta$, то есть отрывов:

$$
J_{\xi} \ddot{\psi}_{j}=b F_{j \eta}, \quad j=1,2 .
$$

Таким образом, мы получили замкнутую систему 10 уравнений (1.6), (1.7), (1.9), (1.10) для определения 10 неизвестных: $v, \varphi, \psi_{1}, \psi_{2}, N_{1}, N_{2}, F_{1 \xi}, F_{2 \xi}, F_{1 \eta}, F_{2 \eta}$. Отметим, что $\psi_{1}$ и $\psi_{2}$ являются циклическими переменными (решение зависит только от их производных).

Задача, которую мы будем в дальнейшем рассматривать, заключается в следующем. Пусть заданы начальные условия:

$$
\left\{\mathbf{v}_{C}(0), \varphi(0), \dot{\varphi}(0), \dot{\psi}_{1}(0), \dot{\psi}_{2}(0)\right\}
$$

такие, что удовлетворены равенства (1.5) и (1.6) при $t=0$ (то есть в начальный момент мы «запускаем» колесную пару по плоскости так, что не нарушаются условия неголономной (кинематической) связи). Требуется определить решение системы (1.6),(1.7),(1.9),(1.10), которое удовлетворяет условию безотрывности: $N_{j}>0, j=1,2$. В более точной постановке, если задан коэффициент трения $f$ плоскости с колесами, требуется также в процессе движения удовлетворить неравенствам Кулона:

$$
F_{j \xi}^{2}+F_{j \eta}^{2} \leqslant f^{2} N_{j}^{2}, \quad j=1,2 .
$$

Такого рода задача рассматривалась для саней Чаплыгина на наклонной плоскости с трением в работе [5].

Приступим к решению поставленной задачи. Отметим, что в уравнения движения неизвестные $F_{1 \xi}, F_{2 \xi}$ входят только в виде суммы $F_{\xi}=F_{1 \xi}+F_{2 \xi}$, так что на самом деле неизвестных всего девять. Однако, как будет видно из дальнейшего решения, независимых уравнений также ровно девять (сумма двух уравнений из (1.10) дает первое уравнение (1.9)!).

Решаем систему (1.6), (1.7), (1.9), (1.10) последовательным исключением неизвестных. 
1. Исключаем из первого уравнения (1.7) и второго уравнения (1.9) неизвестную $F_{\xi}=F_{1 \xi}+$ $F_{2 \xi}$. Получим уравнение:

$$
J_{\xi} \dot{\varphi}\left(\dot{\psi}_{1}+\dot{\psi}_{2}\right)=\left(N_{1}-N_{2}\right) l-b m_{0}(v \dot{\varphi}+g \sin \alpha \sin \varphi) .
$$

2. Исключаем $\dot{\psi}_{1}, \dot{\psi}_{2}, F_{1 \eta}, F_{2 \eta}$ :

$$
F_{1 \eta}=-\frac{J_{\xi}}{b^{2}}(\dot{v}-l \ddot{\varphi}), \quad F_{2 \eta}=-\frac{J_{\xi}}{b^{2}}(\dot{v}+l \ddot{\varphi}) .
$$

В итоге имеем систему:

$$
\left\{\begin{array}{l}
-\dot{\varphi} \cdot J_{\xi} \frac{2 v}{b l}=N_{1}-N_{2}+\frac{b m_{0}}{l}(v \dot{\varphi}+g \sin \alpha \sin \varphi), \\
m_{0} \dot{v}=-\frac{2 J_{\xi}}{b^{2}} \dot{v}+m_{0} g \sin \alpha \sin \varphi \\
0=N_{1}+N_{2}-m_{0} g \cos \alpha \\
\left(J_{0}+J_{\xi} \frac{2 l^{2}}{b^{2}}\right) \ddot{\varphi}=0 .
\end{array}\right.
$$

Мы получили систему четырех уравнений для определения четырех неизвестных: $\varphi, v, N_{1}, N_{2}$. Решения системы (1.11) нетрудно получаются:

$$
\begin{gathered}
\left\{\begin{array}{l}
\dot{\varphi}=\omega_{0}=\text { const }=\dot{\varphi}(0), \\
\dot{v}=k g \sin \alpha \cos \left(\omega_{0} t+\varphi_{0}\right), \quad k=\frac{1}{1+2 \rho^{2} / b^{2}},
\end{array}\right. \\
\left\{\begin{array}{l}
2 N_{1}=m_{0}\left[g \cos \alpha-g \frac{b}{l} \sin \alpha \sin \varphi-\frac{b}{l} v \omega_{0}-\frac{b}{l} v \omega_{0}\left(\frac{2 g^{2}}{b^{2}}\right)\right], \\
2 N_{2}=m_{0}\left[g \cos \alpha+g \frac{b}{l} \sin \alpha \sin \varphi+\frac{b}{l} v \omega_{0}+\frac{b}{l} v \omega_{0}\left(\frac{2 g^{2}}{b^{2}}\right)\right],
\end{array}\right.
\end{gathered}
$$

где $\rho^{2}=J_{\xi} / m_{0}$.

Интегрирование соотношения (1.12) приводит к формуле:

$$
v(t)=v_{0}+\frac{g \sin \alpha}{\omega_{0}\left(1+\frac{2 \rho^{2}}{b^{2}}\right)}\left[\sin \left(\omega_{0} t+\varphi_{0}\right)-\sin \varphi_{0}\right] .
$$

Пусть $x$ - координата центра масс $C$ по линии наибольшего ската (вниз по наклонной плоскости), $y$ - перпендикулярная ей координата, тогда имеем:

$$
\dot{x}=v \cos \varphi, \quad \dot{y}=v \sin \varphi .
$$

Несложное интегрирование последних равенств приводит к выводу, что центр масс движется по циклоиде, перпендикулярной линии наибольшего ската, то есть колесная пара не скатывается вниз.

Исследование формул (1.12), (1.13) приводит к заключению, что безотрывное движение, то есть положительность нормальных реакций $N_{1}, N_{2}$, реализуется при выполнении неравенства:

$$
\frac{\omega_{0} v_{0}}{g}\left(1+\frac{2 \rho^{2}}{b^{2}}\right)<\frac{l}{b} \cos \alpha-2 \sin \alpha+\sin \alpha \sin \varphi_{0} .
$$

Например, при $v_{0}=0, \varphi_{0}=0$ имеем $\operatorname{tg} \alpha<l /(2 b)$, то есть опрокидывание может случиться при достаточно больших $\alpha: \operatorname{tg} \alpha>l /(2 b)$.

Пусть неравенство (1.15) нарушено, тогда обязательно наступит такой момент времени, что произойдет обнуление нормальной реакции, например, $N_{1}$. Итак, пусть $N_{1}\left(t_{1}\right)=0$ и дальнейшее движение возможно только с отрывом колеса 1 (при сохранении контакта колеса 2). Рассмотрим начало такого отрыва, то есть возникающий поворот колесной пары вокруг оси $C \eta$. 


\section{МЕХАНИКА}

2008. Вып. 3

Будем определять такой поворот углом $\chi$, отсчитываемым против часовой стрелки, если смотреть с положительной части оси $C \eta$ (см. рис. 1). Рассматривая дальнейшее движение, будем считать, что $\chi \approx 0, \dot{\chi} \approx 0$, но $\ddot{\chi} \neq 0$. Тогда имеем:

$$
\begin{gathered}
v_{C \xi}=0, \quad v_{C \eta}=v, \quad v_{C \zeta}=l \dot{\chi} \\
K_{C \xi}=J_{\xi}\left(\dot{\psi}_{1}+\dot{\psi}_{2}\right), \quad K_{C \eta}=J_{0} \dot{\chi}, \quad K_{C \zeta}=J_{0} \dot{\varphi}, \\
\boldsymbol{\omega}_{0}=(0, \dot{\chi}, \dot{\varphi}) .
\end{gathered}
$$

Тогда уравнения движения (1.7), (1.9), (1.10) имеют вид:

$$
\begin{gathered}
m_{0}(-v \dot{\varphi})=F_{2 \xi}+m_{0} g \sin \alpha \sin \varphi, \\
m_{0} \dot{v}=F_{2 \eta}+m_{0} g \sin \alpha \cos \varphi, \\
m_{0}(\ddot{\chi} l)=N_{2}-m_{0} g \cos \alpha, \\
J_{\xi} \ddot{\psi}_{2}=b F_{2 \eta}, \\
J_{0} \ddot{\chi}+J_{\xi} \dot{\varphi}\left(\dot{\psi}_{1}+\dot{\psi}_{2}\right)=-N_{2} l-b F_{2 \xi}, \\
J_{0} \ddot{\varphi}=l F_{2 \eta}, \\
J_{\xi} \ddot{\psi}_{1}=0, \quad J \ddot{\psi}_{2}=b F_{2 \eta} .
\end{gathered}
$$

Кроме того, (1.6) превратится в одну кинематическую связь:

$$
v+l \dot{\varphi}=-b \dot{\psi}_{2} \rightarrow \dot{v}+l \ddot{\varphi}=-b \ddot{\psi}_{2} .
$$

Последовательно исключая неизвестные, $F_{2 \xi}, F_{2 \eta}, N_{2}, \dot{\psi}_{1}, \dot{\psi}_{2}$, мы получим три уравнения:

$$
\begin{gathered}
\dot{v}\left(m_{0}+m_{0} \frac{\rho^{2}}{b^{2}}\right)+m_{0} \frac{\rho^{2}}{b^{2}} l \ddot{\varphi}=m_{0} g \sin \alpha \cos \varphi, \\
\ddot{\chi}\left(J_{0}+m_{0} l^{2}\right)=\frac{2 v \dot{\varphi}}{b} J_{\xi}+b m_{0} v \dot{\varphi}+m_{0} g(b \sin \alpha \sin \varphi-l \cos \alpha), \\
J_{0} \ddot{\varphi}-m_{0} l \dot{v}=-m_{0} g l \sin \alpha \cos \varphi
\end{gathered}
$$

для определения трех неизвестных $\varphi, v, \chi$.

Учтем теперь, что в момент отрыва $N_{1}=0$, то есть, в соответствии с (1.13), имеем:

$$
g \cos \alpha-g \frac{b}{l} \sin \alpha \sin \varphi-\frac{b}{l} v \omega_{0}-\frac{b}{l} v \omega_{0} \frac{2 \rho^{2}}{b^{2}}=0 .
$$

Тогда и правая часть равенства (1.17) будет равна нулю, то есть $\ddot{\chi}=0$. Дифференцируя (1.17), вычисляем $\dddot{\chi}$ :

$$
\left(J_{0}+m_{0} l^{2}\right) \dddot{\chi}=\frac{2 \dot{v} \dot{\varphi}}{b} J_{\xi}+\frac{2 v}{b} J_{\xi} \ddot{\varphi}+b m_{0}(\dot{v} \dot{\varphi}+v \ddot{\varphi})+m_{0} g b \sin \alpha \cos \varphi \dot{\varphi} .
$$

Из (1.16) и (1.18) находим:

$$
\dot{\varphi}=-\frac{\rho^{2} g l \sin \alpha \cos \varphi}{\rho^{2} \rho_{0}^{2}+\rho_{0}^{2} b^{2}+\rho^{2} l^{2}}, \quad \dot{v}=\frac{\left(\rho_{0}^{2} b^{2}+\rho^{2} l^{2}\right) g \sin \alpha \cos \varphi}{\rho^{2} \rho_{0}^{2}+\rho_{0}^{2} b^{2}+\rho^{2} l^{2}},
$$

где $\rho^{2}=J_{\xi} / m_{0}, \rho_{0}^{2}=J_{0} / m_{0}$.

Подставляя полученные выражения для $\ddot{\varphi}$ и $\dot{v}$ в (1.19), находим

$$
\begin{gathered}
\left(\frac{J_{0}+m_{0} l^{2}}{m_{0}}\right) \dddot{\chi}=\dot{v} \dot{\varphi}\left(b+\frac{2 \rho^{2}}{b}\right)+v \ddot{\varphi}\left(b+\frac{2 \rho^{2}}{b}\right)+g b \sin \alpha \cos \varphi \dot{\varphi}= \\
=b g \sin \alpha \cos \varphi\left[\left(1+\frac{2 \rho^{2}}{b}\right)\left(\dot{\varphi} \frac{\rho_{0}^{2} b^{2}+\rho^{2} l^{2}}{\rho^{2} \rho_{0}^{2}+\rho_{0}^{2} b^{2}+\rho^{2} l^{2}}-v \frac{\rho^{2} l}{\rho^{2} \rho_{0}^{2}+\rho_{0}^{2} b^{2}+\rho^{2} l^{2}}\right)+\dot{\varphi}\right]= \\
=b g \sin \alpha \cos \varphi\left[\left(1+\frac{2 \rho^{2}}{b}\right) \frac{\dot{\varphi}\left(\rho_{0}^{2} b^{2}+\rho^{2} l^{2}\right)-v \rho^{2} l}{\rho^{2} \rho_{0}^{2}+\rho_{0}^{2} b^{2}+\rho^{2} l^{2}}+\dot{\varphi}\right],
\end{gathered}
$$

где $\rho^{2}=\frac{J_{\xi}}{m_{0}}, \rho_{0}^{2}=\frac{J_{0}}{m_{0}}$. 
МЕХАНИКА

Возьмем, к примеру, такие начальные условия:

$$
v_{0}=0, \quad \varphi_{0}=0, \quad \dot{\varphi}(0)=\omega_{0} \neq 0 .
$$

Тогда имеем, в соответствии с формулой (1.14):

$$
v(t)=\frac{g \sin \alpha}{\omega_{0}\left(1+\frac{2 \rho^{2}}{b}\right)} \sin \left(\omega_{0} t\right) .
$$

Для нормальной реакции $N_{1}$ из (1.13) получим:

$$
\frac{2}{m_{0}} N_{1}=g \cos \alpha-2 g \frac{b}{l} \sin \alpha \sin \left(\omega_{0} t\right)=g \cos \alpha\left(1-\frac{2 b}{l} \operatorname{tg} \alpha \sin \omega_{0} t\right) .
$$

Обнуление $N_{1}$ происходит первый раз при $t=t_{1}$, таком, что:

$$
\sin \left(\omega_{0} t_{1}\right)=\frac{l}{2 b \operatorname{tg} \alpha} \quad\left(\operatorname{tg} \alpha>\frac{l}{2 b} !\right) .
$$

Тогда при $t=t_{1}$ имеем:

$$
v\left(t_{1}\right)=\frac{g \cos \alpha}{\omega_{0}\left(1+\frac{2 \rho^{2}}{b}\right)} \frac{l}{2 b} .
$$

Ясно, что, выбирая $\omega_{0}$ достаточно малым, мы можем добиться больших значений $v\left(t_{1}\right)$, а формула (1.20) нам тогда дает $\dddot{\chi}<0$, то есть возникает парадоксальная ситуация при отрыве! Эти рассуждения приводят нас к выводу, что, в случаях когда могут быть обнуления нормальной реакции (нарушение неравенства (1.15)), необходимо учитывать конечный коэффициент трения и рассматривать процесс движения колесной пары с возможными переходами к ситуации проскальзывания колес, что, конечно, усложняет решение задачи.

Замечание 1. Нетрудно обобщить решение задачи на тот случай, когда к колесной паре приложены другие внешние силы (помимо силы тяжести). Например, тяговая сила (колесная пара - автомобильный прицеп) или управляющий момент на оси $O_{1} O_{2}$ (колесная пара автомобиля или робот-тележка). Отметим, что рассматриваемая задача изучалась еще классиками теоретической механики [6].

\section{§ 2. Динамика плоской колесной модели (скейтборд)}

На рис. 2 изображена модель скейтборда в плане (то есть вид сверху). Это - две колесные пары $A_{1} A_{2}, B_{1} B_{2}$, соединенные в своих центрах масс жестким стержнем $A B$. Шарниры в соединениях $A$ и $B$ предполагаются идеальными и цилиндрическими, а качение колес $A_{1}, A_{2}, B_{1}, B_{2}$ по опорной плоскости происходит без проскальзывания, то есть наложена неголономная связь.

В данной модели мы предполагаем (так же как и в [1-4]), что эта связь реализуется только поперечными силами $\mathbf{F}_{A}$ и $\mathbf{F}_{B}$, приложенными соответственно к $A_{1} A_{2}$ и $B_{1} B_{2}$ (см. рис. 2). Учет продольных сил рассматривался, например, в статье [7].

Наличие неголономной связи дает право считать, что скорости центров масс колесных пар, обозначаемых как $\mathbf{v}_{A}$ и $\mathbf{v}_{B}$, направлены всегда перпендикулярно соответствующим осям, то есть $\mathbf{v}_{A} \perp A_{1} A_{2}, \quad \mathbf{v}_{B} \perp B_{1} B_{2}$ (см. рис. 2).

Далее введены следующие обозначения: $\theta_{1}, \theta_{2}$ - углы отклонения колесных пар $A_{1} A_{2}, B_{1} B_{2}$ от продольной оси $\xi$ скейтборда, причем $\theta_{1}$ отсчитываем по часовой стрелке, а $\theta_{2}-$ против оной; $A C=a, C B=b, C$ - центр масс всей системы, который мы предполагаем неизменным, то есть $a=$ const, $b=$ const ; $\varphi$ - угол поворота стержня $A B$ относительно неизменно ориентированной оси $C x$, отсчитываемый против часовой стрелки; $P$ - мгновенный центр скоростей стержня $A B$, который, в силу неголономных связей, является пересечением прямых $A_{1} A_{2}$ 


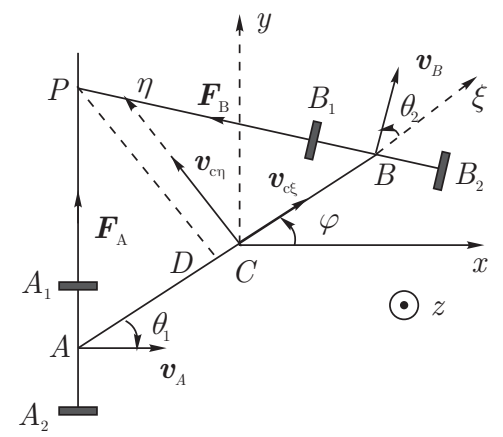

Рис. 2.

и $B_{1} B_{2} ; m_{A B}, m_{A}, m_{B}$ - массы соответственно стержня $A B$, колесных пар $A_{1} A_{2}, B_{1} B_{2}$; $J_{A B}$ - момент инерции стержня $A B$ - вместе с сосредоточенными массами $m_{A}$ и $m_{B}$ на его концах относительно точки $C ; J_{A}, J_{B}$ - моменты инерции колесных пар соответственно $A_{1} A_{2}, B_{1} B_{2}$ относительно собственных центров масс $A$ и $B ; C \xi \eta$ - система координат, жестко связанная со стержнем $A B$.

Для трех углов $\varphi, \theta_{1}, \theta_{2}$ мы можем составить три уравнения динамики, которые не содержат неизвестных сил $F_{1}, F_{B}$ и сил реакций в шарнирах $A$ и $B$ :

$$
\begin{gathered}
K_{P z}+m\left[\mathbf{v}_{p} \times \mathbf{v}_{c}\right]_{z}=0, \\
J_{A}\left(\ddot{\varphi}-\ddot{\theta}_{1}\right)=M_{A z}, \\
J_{B}\left(\ddot{\varphi}+\ddot{\theta}_{2}\right)=M_{B z} .
\end{gathered}
$$

Уравнение (2.1) - это уравнение кинетического момента относительно подвижной точки $P$ (см. [8]); а уравнения (2.2) и (2.3) - уравнения кинетических моментов колесных пар относительно их собственных центров масс. В этих уравнениях введены обозначения: $m=$ $m_{A B}+m_{A}+m_{B}$ - общая масса системы; $K_{P z}$ - кинетический момент системы относительно точки $P, \mathbf{v}_{p}$ - скорость точки $P$ (как геометрической относительно неподвижной системы координат), $\mathbf{v}_{c}-$ скорость центра масс $C ; M_{A z}, M_{B z}$ - управляющие моменты в шарнирах $A$ и $B$ (если таковые имеются). Пусть $D$ - проекция точки $P$ на стержень $A B$ (см. рис. 2). Обозначим $C D=\xi_{0}$. Тогда имеем, в соответствии с теоремой Кенига (см. [9]):

$$
\begin{gathered}
K_{P z}=K_{c z}+m\left(v_{c \xi} \cdot P D+v_{c \eta} \cdot C D\right) ; \\
K_{c z}=J_{A B} \dot{\varphi}+J_{B}\left(\dot{\varphi}+\dot{\theta}_{2}\right)+J_{A}\left(\dot{\varphi}-\theta_{1}\right) .
\end{gathered}
$$

Далее, из кинематических и геометрических соображений получаем:

$$
\begin{gathered}
{\left[\mathbf{v}_{p} \times \mathbf{v}_{c}\right]_{z}=v_{p \xi} v_{c \eta}-v_{p \eta} v_{c \xi}, \quad v_{c \xi}=\dot{\varphi} \cdot P D, \quad v_{c \eta}=\dot{\varphi} \cdot C D,} \\
P D=\left(a-\xi_{0}\right) \operatorname{ctg} \theta_{1}=\left(b+\xi_{0}\right) \operatorname{ctg} \theta_{2} \quad\left(\text { где } \xi_{0}=C D\right) . \quad \text { Тогда } \\
\xi_{0}=C D=\frac{a \operatorname{tg} \theta_{2}-b \operatorname{tg} \theta_{1}}{\operatorname{tg} \theta_{1}+\operatorname{tg} \theta_{2}}, \quad P D=\frac{a+b}{\operatorname{tg} \theta_{1}+\operatorname{tg} \theta_{2}} .
\end{gathered}
$$

Абсолютная скорость точки $P$, в соответствии с теоремой о сложении скоростей при сложном движении (см. [9]):

$$
\mathbf{v}_{p}=\mathbf{v}_{p}^{(r)}+\mathbf{v}_{p}^{(e)}=\mathbf{v}_{p}^{(r)}+\mathbf{v}_{c}+\mathbf{v}_{p c}^{(e)} .
$$

Но так как $P-$ мгновенный центр скоростей, то $\mathbf{v}_{p}^{(e)}=\mathbf{v}_{c}+\mathbf{v}_{p c}^{(e)}$. Тогда получаем: $v_{p \xi}=$ $-(C D)^{*}, v_{p \eta}=-(P D)^{\prime}$, то есть:

$$
\begin{aligned}
& v_{p \xi}=\frac{a+b}{\left(\operatorname{tg} \theta_{1}+\operatorname{tg} \theta_{2}\right)^{2}}\left(\frac{\theta_{1} \operatorname{tg} \theta_{2}}{\cos ^{2} \theta_{1}}-\frac{\theta_{2} \operatorname{tg} \theta_{1}}{\cos ^{2} \theta_{2}}\right), \\
& v_{p \eta}=-\frac{a+b}{\left(\operatorname{tg} \theta_{1}+\operatorname{tg} \theta_{2}\right)^{2}}\left(\frac{\theta_{1}}{\cos ^{2} \theta_{1}}+\frac{\theta_{2}}{\cos ^{2} \theta_{2}}\right) .
\end{aligned}
$$


Используя приведенные формулы, мы получим следующие соотношения:

$$
\begin{aligned}
& {\left[\mathbf{v}_{p} \times \mathbf{v}_{c}\right]_{z}=} \frac{(a+b) \dot{\varphi}}{\left(\operatorname{tg} \theta_{1}+\operatorname{tg} \theta_{2}\right)^{3}}\left[\frac{\theta_{1}}{\cos ^{2} \theta_{1}}\left(a \operatorname{tg}^{2} \theta_{2}-b \operatorname{tg} \theta_{1} \operatorname{tg} \theta_{2}+a+b\right)+\right. \\
&\left.+\frac{\theta_{2}}{\cos ^{2} \theta_{2}}\left(-a \operatorname{tg} \theta_{1} \operatorname{tg} \theta_{2}+b \operatorname{tg}^{2} \theta_{1}+a+b\right)\right], \\
& K_{p z}=K_{c z}+\frac{m \ddot{\varphi}}{\left(\operatorname{tg} \theta_{1}+\operatorname{tg} \theta_{2}\right)^{2}}\left[(a+b)^{2}+\left(a \operatorname{tg} \theta_{2}-b \operatorname{tg} \theta_{1}\right)^{2}\right]+ \\
&+\frac{2 m \dot{\varphi}(a+b)}{\left(\operatorname{tg} \theta_{1}+\operatorname{tg} \theta_{2}\right)^{3}}\left[-\frac{\theta_{2}}{\cos ^{2} \theta_{2}}\left(-a \operatorname{tg} \theta_{1} \operatorname{tg} \theta_{2}+b \operatorname{tg}^{2} \theta_{1}+a+b\right)-\right. \\
&\left.\quad-\frac{\theta_{1}}{\cos ^{2} \theta_{1}}\left(a \operatorname{tg}^{2} \theta_{2}-b \operatorname{tg} \theta_{1} \operatorname{tg} \theta_{2}+a+b\right)\right], \\
& K_{c z}^{*}=J_{A B} \ddot{\varphi}+J_{B}\left(\ddot{\varphi}+\ddot{\theta}_{2}\right)+J_{A}\left(\ddot{\varphi}-\ddot{\theta}_{1}\right)=J_{A B} \ddot{\varphi}+M_{B z}+M_{A z} .
\end{aligned}
$$

Таким образом, уравнение (2.1) принимает вид:

$$
\begin{gathered}
\ddot{\varphi}\left\{J_{A B}+\frac{m}{\left(\operatorname{tg} \theta_{1}+\operatorname{tg} \theta_{2}\right)^{2}}\left[(a+b)^{2}+\left(a \operatorname{tg} \theta_{2}-b \operatorname{tg} \theta_{1}\right)^{2}\right]\right\}- \\
-\frac{m \dot{\varphi}(a+b)}{\left(\operatorname{tg} \theta_{1}+\operatorname{tg} \theta_{2}\right)^{3}}\left[\frac{\theta_{2}}{\cos ^{2} \theta_{2}}\left(a+b-a \operatorname{tg} \theta_{1} \operatorname{tg} \theta_{2}+b \operatorname{tg}^{2} \theta_{1}\right)+\right. \\
\left.+\frac{\theta_{1}}{\cos ^{2} \theta_{1}}\left(a+b+a \operatorname{tg}^{2} \theta_{2}-b \operatorname{tg} \theta_{1} \operatorname{tg} \theta_{2}\right)\right]=-M_{A z}-M_{B z} .
\end{gathered}
$$

Уравнения (2.2), (2.3), (2.4) образуют замкнутую (относительно $\left.\varphi, \theta_{1}, \theta_{2}\right)$ систему уравнений.

Рассмотрим частные случаи.

$1^{\circ}$. Закрепим колесную пару $A_{1} A_{2}$ жестко, то есть полагаем $\theta_{1} \equiv 0 \Rightarrow M_{A z}=J_{A} \ddot{\varphi}$. Тогда уравнение (2.4) будет иметь вид:

$$
\begin{gathered}
\ddot{\varphi}\left\{J_{A B}+J_{A}+\frac{m}{\operatorname{tg}^{2} \theta_{2}}\left[(a+b)^{2}+a^{2} \operatorname{tg}^{2} \theta_{2}\right]\right\}- \\
-\frac{m(a+b) \dot{\varphi}}{\operatorname{tg}^{3} \theta_{2}}\left[\frac{\theta_{2}}{\cos ^{2} \theta_{2}}(a+b)\right]=-M_{B z},
\end{gathered}
$$

получаем модель $[1,4]$.

$2^{\circ}$. Управление скейтбордом, то есть $M_{A z}$ и $M_{B z}$ подбираем так, что $\theta_{1}=\theta_{2}=\theta$ (одинаковые отклонения колесных пар, но в разные стороны). Тогда из (2.2), (2.3) мы получаем:

$$
M_{A z}+M_{B z}=\left(J_{A}+J_{B}\right) \ddot{\varphi} .
$$

А из уравнения (2.4) имеем:

$$
\begin{gathered}
\ddot{\varphi}\left\{J_{A B}+J_{A}+J_{B}+\frac{m}{4 \operatorname{tg}^{2} \theta}\left[(a+b)^{2}+\operatorname{tg}^{2} \theta(a-b)^{2}\right]\right\}- \\
-\frac{m \dot{\varphi}(a+b)}{4 \operatorname{tg}^{3} \theta}\left[\frac{\theta}{\cos ^{2} \theta}(a+b)\right]=0,
\end{gathered}
$$

то есть получаем модель из [3].

Отметим, что для рассматриваемой модели в случае отсутствия управляющих моментов $M_{A z}, M_{B z}$ (или их консервативности) будет справедлив интеграл энергии. Вычислим кинетическую энергию системы:

$$
T=\frac{m}{2}\left(v_{c \xi}^{2}+v_{c \eta}^{2}\right)+\frac{J_{A B}}{2} \dot{\varphi}^{2}+\frac{J_{A}}{2}\left(\dot{\varphi}-\dot{\theta}_{1}\right)^{2}+\frac{J_{B}}{2}\left(\dot{\varphi}+\dot{\theta}_{2}\right)^{2},
$$




\section{МЕХАНИКА}

последние два слагаемых суть константы (при $M_{A z}=M_{B z}=0$ ). Поэтому интеграл энергии при $M_{A z}=M_{B z}=0$ принимает вид:

$$
m\left(v_{c \xi}^{2}+v_{c \eta}^{2}\right)+J_{A B} \dot{\varphi}^{2}=h_{0}^{2}=\text { const. }
$$

Из полученных выше кинематических формул следует:

$$
v_{c \xi}=\frac{a+b}{\operatorname{tg} \theta_{1}+\operatorname{tg} \theta_{2}} \cdot \dot{\varphi}, \quad v_{c \eta}=\frac{a \operatorname{tg} \theta_{2}-b \operatorname{tg} \theta_{1}}{\operatorname{tg} \theta_{1}+\operatorname{tg} \theta_{2}} \cdot \dot{\varphi},
$$

и тогда интеграл энергии приобретает вид:

$$
2 T=\dot{\varphi}^{2}\left[J_{A B}+m \frac{(a+b)^{2}+\left(a \operatorname{tg} \theta_{2}-b \operatorname{tg} \theta_{1}\right)^{2}}{\left(\operatorname{tg} \theta_{1}+\operatorname{tg} \theta_{2}\right)^{2}}\right]=h_{0}^{2}=\text { const. }
$$

Из уравнений (2.2), (2.3) при $M_{A z}=M_{B z}=0$ мы получим:

$$
\theta_{1}=\varphi-h_{1} t+p_{1}, \quad \theta_{2}=-\varphi+h_{2} t+p_{2},
$$

где $h_{1}, p_{1}, h_{2}, p_{2}$ - константы, определяемые начальными условиями. Подставляя (2.9) в (2.8), мы получаем для определения $\varphi$ нестационарное уравнение 1-го порядка.

$1^{\circ}$. Рассмотрим частный случай: закрепленную колесную пару $A_{1} A_{2}$, то есть $\theta_{1} \equiv 0$, тогда интеграл энергии (2.8) имеет вид

$$
\begin{aligned}
& \dot{\varphi}^{2}\left[J_{A B}+m \frac{(a+b)^{2}+a^{2} \operatorname{tg}^{2} \theta_{2}}{\operatorname{tg}^{2} \theta_{2}}\right]=h_{0}^{2} \Rightarrow \\
& \dot{\varphi}^{2}\left[\left(J_{A B}+m a^{2}\right)+m(a+b)^{2} \operatorname{ctg}^{2} \theta_{2}\right]=h_{0}^{2} .
\end{aligned}
$$

Уравнение (2.3) при $M_{B z}=0$ дает:

$$
\dot{\varphi}=\omega_{0}-\theta_{2},
$$

и из (2.10) мы получаем, обозначая $A^{2}=J_{A B}+m a^{2}, B^{2}=m(a+b)^{2}, \lambda=h_{0} / \omega_{0}, x=\operatorname{tg} \theta_{2}$ :

$$
\begin{gathered}
\frac{d \theta_{2}}{d t}=\frac{\omega_{0} \sqrt{B^{2}+A^{2} \operatorname{tg}^{2} \theta_{2}}-h_{0} \operatorname{tg} \theta_{2}}{\sqrt{B^{2}+A^{2} \operatorname{tg}^{2} \theta_{2}}} \rightarrow \\
I=\int \frac{d \theta_{2} \sqrt{B^{2}+A^{2} \operatorname{tg}^{2} \theta_{2}}}{\sqrt{B^{2}+A^{2} \operatorname{tg}^{2} \theta_{2}}-\lambda \operatorname{tg} \theta_{2}}=\omega_{0} t+C .
\end{gathered}
$$

Обозначая $x=\operatorname{tg} \theta_{2}$, мы получаем

$$
I=\int \frac{d x}{1+x^{2}}+\lambda \int \frac{x d x \sqrt{B^{2}+A^{2} \operatorname{tg}^{2} \theta_{2}}}{\left(1+x^{2}\right)\left[B^{2}+\left(A^{2}-\lambda^{2}\right) x^{2}\right]}+\lambda^{2} \int \frac{x^{2} d x}{\left(1+x^{2}\right)\left[B^{2}+\left(A^{2}-\lambda^{2}\right) x^{2}\right]} .
$$

Нетрудно показать что все эти интегралы берутся в элементарных функциях.

Таким образом, в указанном случае уравнения движения системы интегрируются в функциях времени при любых значениях параметров и начальных условилх.

$2^{\circ} . \theta_{1}=\theta_{2}=\theta$ (скейтборд управляемый $) \Rightarrow$

$$
\begin{gathered}
\dot{\varphi}\left\{J_{A B}+J_{A}+J_{B}+\frac{m}{4}\left[\frac{(a+b)^{2}}{\operatorname{tg}^{2} \theta}+(a-b)^{2}\right]\right\}-\frac{m \dot{\varphi}(a+b)^{2}}{4 \operatorname{tg}^{3} \theta} \cdot \frac{\theta}{\cos ^{2} \theta}=0 ; \\
\ddot{\varphi}=\frac{1}{2} \frac{d\left(\dot{\varphi}^{2}\right)}{d \varphi} ; \quad \dot{\varphi} \theta=(\dot{\varphi})^{2} \frac{d \theta}{d \varphi} ; \quad A=\frac{m}{4}(a-b)^{2}+J_{A B}+J_{A}+J_{B} ; \\
d\left(\dot{\varphi}^{2}\right)\left[A+\frac{m(a+b)^{2} \operatorname{ctg}^{2} \theta}{4}\right]-\frac{m(a+b)^{2}}{2 \operatorname{tg}^{3} \theta \cos ^{2} \theta} \cdot(\dot{\varphi})^{2} d \theta=0 \rightarrow \\
\frac{d\left(\dot{\varphi}^{2}\right)}{\dot{\varphi}^{2}}=d \theta \cdot \frac{m(a+b)^{2}}{2 \operatorname{tg}^{3} \theta \cos ^{2} \theta\left[A+\frac{m(a+b)^{2} \operatorname{ctg}^{2} \theta}{4}\right]} .
\end{gathered}
$$


Обозначаем $\dot{\varphi}^{2}=u ; \operatorname{tg} \theta=v$; тогда:

$$
\begin{aligned}
& \frac{d u}{u}=\frac{m(a+b)^{2}}{2} \cdot \frac{d v}{A v^{3}+\frac{m(a+b)^{2}}{4} v} ; \quad \frac{m(a+b)^{2}}{4}=B ; \\
& \ln u-C_{0}=\frac{m(a+b)^{2}}{2} \int \frac{d v}{v\left(A v^{2}+\frac{m(a+b)^{2}}{4}\right)}=2 B \int \frac{d v}{v\left(A v^{2}+B\right)} \text {; } \\
& \ln u-C_{0}=\ln \frac{v^{2}}{A v^{2}+B} \Rightarrow \frac{u\left(A v^{2}+B\right)}{v^{2}}=C_{0}=\text { const; } \\
& \int \frac{d v}{v\left(A v^{2}+B\right)}=\int\left(\frac{C_{0}}{v}+\frac{D_{0} v+F_{0}}{A v^{2}+B}\right) d v ; \\
& C_{0}\left(A v^{2}+B\right)+D_{0} v^{2}+E_{0} v=1 \quad \rightarrow \\
& C_{0} B=1 ; \quad E_{0}=0 ; \quad C_{0} A+D_{0}=0 ; \\
& C_{0}=\frac{1}{B} ; \quad D_{0}=-\frac{A}{B} ; \\
& \int \frac{d v}{v\left(A v^{2}+B\right)}=\int \frac{1 / B}{v} d v-\frac{2 A}{2 B} \int \frac{v d v}{a v^{2}+B}= \\
& =\frac{1}{B} \ln v-\frac{1}{2 B} \ln \left(A v^{2}+B\right)=\frac{1}{B} \ln \frac{v}{\sqrt{A v^{2}+B}} ; \\
& \dot{\varphi}^{2} \frac{A \operatorname{tg}^{2} \theta+B}{\operatorname{tg}^{2} \theta}=C_{0} \Rightarrow \dot{\varphi}=\frac{C_{0} \operatorname{tg} \theta}{\sqrt{A \operatorname{tg}^{2} \theta+B}} .
\end{aligned}
$$

Полученное соотношение является определяющим уравнением движения скейтборда.

\section{СПИСОК ЛИТЕРАТУРЫ}

1. Мартыненко Ю. Г. К теории обобщенного эффекта Магнуса для неголономных механических систем // ПММ. - 2004. - Т. 68, № 6. - С. 948-957.

2. Kuleshov A.S. Further Development of the Mathematical Model of a Snakeboard // Regul. \& Chaotic Dyn. - 2007. - Vol. 12, No. 3. - P. 321-334.

3. Ispolov Yu. G., Smolnikov B. A. Skateboard dynamics // Computer Methods in Appl. Mech. and Eng. 1996. - № 131. - Р. 327-333.

4. Лобас Л. Г. Неголономные модели колесных экипажей. - Киев: Наукова думка, 1986 - 232 с.

5. Ж Журавлев В.Ф., Фуфаев Н. А. Механика систем с неудерживающими связями. Отв. редактор академик РАН Д.М. Климов. - М.: Наука, Институт проблем механики РАН, 1993. - 240 с.

6. Раус Э. Дж. Динамика системы твердых тел. Т. 2. - М.: Наука, 1983. - 544 с.

7. Буданов В.М., Девянин Е.А. О движении колесных роботов // ПММ. - 2003. - Т. 67, № 2. C. 244-255.

8. Суслов Г.К. Теоретическая механика. Издание третье посмертное. Под ред. проф. Н.Н. Бухгольца и В.К. Гольцмана. - М.-Л.: ОГИЗ ГИТТЛ, 1944. - 655 с.

9. Маркеев А.П. Теоретическая механика. Изд. 4-е, испр. - Москва-Ижевск. - Регулярная и хаотическая динамика, 2007. - 591 с.

Поступила в редакцию 21.11.08

\section{G. M. Rozenblat \\ Dynamics of non-holonomic models of wheel carriage}

We consider two problems of non-holonomic mechanics: 1) motion of a wheel pair without sliding (two disks freely fixed on the axle) on the inclined plane in gravitational field and 2) motion of a plain wheel model such as skateboard

Keywords: non-holonomic constraints, basic theorems of dynamics, wheel carriage, skateboard.

Mathematical Subject Classifications: 37N15

Розенблат Григорий Маркович, к.ф.-м.н., доцент кафедры теоретической механики, Московский автомобильно-дорожный институт, 125319, Москва, Ленинградский проспект, 64, E-mail: gr51@mail.ru 